\title{
Repercussões no neonato da utilização de redes de descanso e posição prono
}

\author{
Repercussions in newborns using hammocks and prone position
}

\begin{abstract}
Claudenilksan Margarida Borges de Queiroz ${ }^{1}$, Aldair Darlan Santos-de-Araújo ${ }^{1}$, Lília Maria Ferreira Silva ${ }^{1}$, José Augusto Gomes Silva Júnior ${ }^{1}$, Daniela Bassi ${ }^{2}$, Cícera Trindade Santos de Souza ${ }^{3}$, Alice Sá Carneiro Ribeiro ${ }^{3}$, Ana Carolina do Nascimento Calles ${ }^{3}$
\end{abstract}

\begin{abstract}
Resumo: Um posicionamento adequado influencia o desenvolvimento neurossensorial, proporcionando conforto, além de auxiliar na melhora da função respiratória de um recém-nascido (RN), o que por sua vez, também reduz a sensação dolorosa. $\mathrm{O}$ objetivo desta pesquisa consiste em verificar a utilização das redes de descanso e do posicionamento em prono no alívio da dor e no comportamento dos sinais vitais em recém-nascidos pré-termo (RCPT). Trata-se de pesquisa intervencionista com uma amostra de 20 recémnascidos pré-termos internados na Unidade de Terapia Intensiva Neonatal (UTIN) do Hospital do Açúcar na cidade de Maceió (AL, Brasil). Avaliaram-se os sinais vitais e o escore de dor (escala NFCS - Sistema de Codificação da Atividade Facial Neonatal) antes e após os posicionamentos propostos. Esta pesquisa teve os seguintes resultados: ao comparar os valores iniciais e finais para cada variável entre as intervenções prono e redes de descanso, foi observada melhora significativa nos sinais vitais e na dor em ambos os posicionamentos, mas não houve significância estatística $(p>0,05)$ entre as intervenções, indicando que as mesmas causaram alterações semelhantes nos recém-nascidos. Os achados apontam a importância das mudanças de decúbito citados em RNPT estáveis internados em UTIN por se tratarem de métodos não invasivos e de baixo custo que proporcionam benefícios para o desenvolvimento como um todo desses RN.
\end{abstract}

Palavras-Chave: recém-nascido; posicionamento; unidade de terapia intensiva neonatal.

\begin{abstract}
Adequate positioning influences the sensorineural development, providing comfort, besides helping to improve the respiratory function of a newborn (NB), which in turn, also reduces the pain sensation. The aim of this research is to verify the use of rest nets and prone positioning in pain relief and the behavior of vital signs in preterm newborns (RCPT). This is an interventionist study with a sample of 20 preterm newborns admitted to the Neonatal Intensive Care Unit (NICU) of the Hospital do Açúcar in the city of Maceió (AL, Brazil). Vital signs and pain score (NFCS - Neonatal Facial Coding System scale) were evaluated before and after the proposed positioning. This research had the following results: when comparing the initial and final values for each variable between the prone interventions and rest nets, a significant improvement in vital signs and pain was observed in both placements, but there was no statistical significance $(p>0.05)$ among interventions, indicating that they have caused similar changes in newborns. The findings warn the importance of cited decubitus changes in stable preterm NB admitted in the NICU because they are not invasive and inexpensive and that they provide benefits in the development as a whole of these NB.
\end{abstract}

Keywords: newborn; positioning; neonatal intensive care unit.

\footnotetext{
${ }^{1}$ Discente do Curso de Fisioterapia do Centro Universitário Tiradentes, Maceió - AL, Brasil

${ }^{2}$ Docente do Curso de Fisioterapia da Universidade Ceuma, São Luís - MA, Brasil

${ }^{3}$ Docente do Curso de Fisioterapia do Centro Universitário Tiradentes, Maceió - AL, Brasil
}

\section{Autor Correspondente}

Daniela Bassi. Rua Josué Montello, 1 - Jardim Renascença II. CEP: 65075-120. São Luís, Maranhão

Telefone: +559832144277 . E-mail: danielabassifisio@gmail.com 


\section{Introdução}

A neonatologia vem ganhando grande espaço nas últimas décadas, tanto no que se diz respeito à tecnologia quanto no campo cientifico, ocasionando no aumento da sobrevida do recémnascido pré-termo (RNPT) devido às melhorias em seu cuidado ${ }^{1}$. O nascimento prematuro está relacionado com privações da vida intrauterina levando ao fim da gestação antes das 37 semanas devido a fatores como: gemelaridade, hipertensão, tabagismo, reprodução assistida, consumo de álcool e desnutrição ${ }^{2}$.

Os RNPT geralmente são acometidos por uma série de disfunções e doenças, destacando-se entre elas: as alterações respiratórias, cardiovasculares, metabólicas e doenças congênitas, resultando na necessidade do suporte oferecido pela unidade de terapia intensiva neonatal (UTIN) a fim de minimizar os efeitos dos fatores de risco ${ }^{1}$.

De acordo com Cordeiro e Costa ${ }^{3}$, cada recém-nascido (RN) é submetido diariamente a cerca de 50 a 150 procedimentos dolorosos durante os cuidados das UTIN, os quais podem ser identificados por meio de sinais de estresse e desorganização, evidenciados por alterações fisiológicas e comportamentais como: choro, rigidez muscular, expressão facial modificada, alterações no sono, na alimentação, na frequência cardíaca, na frequência respiratória, na saturação de oxigênio, na pressão arterial e no quadro clínico como um todo ${ }^{4,5}$.

Têm sido adotadas medidas não farmacológicas nas rotinas da UTIN com intuito de prevenir e minimizar a dor, tais como: sucção não nutritiva, aleitamento materno, glicose oral antes e após os procedimentos dolorosos e tendo como destaque as mudanças de decúbitos, estando esse associado à diminuição da frequência respiratória ${ }^{6,7}$.
Por meio de um posicionamento adequado, pode-se influenciar 0 desenvolvimento neurossensorial, proporcionando conforto, além de auxiliar na melhora da função respiratória de um $\mathrm{RN}$, o que por sua vez também reduz a sensação dolorosa ${ }^{8}$. Para este fim, indica-se o uso de redes de descanso com a finalidade de simular a posição uterina devido a sua natureza flexora e alinhada, proporcionando mãos próximas à boca, além de movimentos suaves contínuos. Tal atitude promove simetria corporal, sensação de aconchego, além de favorecer a estimulação do sistema vestibular, das reações de equilíbrio e de proteção, e a integração sensorial ${ }^{9}$.

Além do uso das redes, o posicionamento em prono também concede melhora na perfusão pulmonar, - que promove estabilidade da caixa torácica resultando na melhora de sua complacência e aumento da pressão abdominal, e otimiza a mecânica da musculatura respiratória, favorecendo, assim, menor frequência cardíaca, maior tempo de sono e padrão respiratório mais estável, bem como diminuição da irritabilidade $^{10-12}$. Ademais, estimula a musculatura antigravitária devido ao contato com a superfície de apoio, levando a um melhor controle postural ${ }^{13}$.

Visto que a UTIN tem sido apontada como um fator determinante na sobrevivência de RNPT, nota-se a necessidade de um cuidado especial em relação aos procedimentos adotados neste ambiente, tornando de suma importância à utilização de técnicas terapêuticas focadas no conforto e no alívio da dor. Neste contexto, o presente estudo se dispõe a verificar a utilização das redes de descanso e do posicionamento em prono no alívio da dor e no comportamento dos sinais vitais em RNPT estáveis. 
Material e Método

O presente estudo foi realizado na UTIN do Hospital do Açúcar na cidade de Maceió (AL, Brasil). Trata-se de uma pesquisa intervencionista com modelo crossover, constituída por uma amostra de $20 \mathrm{RN}$, de ambos os sexos, hospitalizados na UTIN, iniciada após a aprovação do Comitê de Ética em Pesquisa do Centro Universitário Tiradentes, sob protocolo CAEE no 45669815.7.0000.564. A participação no estudo foi validada pelo termo de consentimento livre e esclarecido assinado pelos pais ou responsáveis. Todo o estudo foi realizado entre os meses de maio e agosto de 2015, sob supervisão de um fisioterapeuta que esteve de plantão na UTIN no período vespertino.

Utilizou-se como critérios de inclusão: pré-termos estáveis e desorganizados (dor, agitação, choro excessivo, dificuldade de entrar em um período de sono profundo). E os critérios de exclusão foram: bebês prematuros clinicamente instáveis apresentando problemas neurológicos ou processo infeccioso, necessidade de suporte ventilatório, e bebês com malformações congênitas faciais.

Neste estudo, verificaram-se os sinais vitais como: dor, frequência cardíaca ( $\mathrm{FC})$, frequência respiratória $(\mathrm{FR})$, pressão arterial média (PAM), saturação de oxigênio $\left(\mathrm{SaO}_{2}\right)$ e temperatura corporal (TAX).

A variável dor foi analisada por meio da escala denominada Sistema de Codificação da Atividade Facial Neonatal (NFCS). Esse instrumento possibilita a verificação da presença ou ausência da dor através da análise de oito movimentos faciais: testa franzida, fenda palpebral comprimida, sulco nasolabial aprofundado, lábios entre abertos, boca esticada vertical ou horizontal, língua tensa, protrusão da língua e tremor do queixo. É importante salientar que se atribuiu um ponto para cada movimento, com pontuação máxima de 8 pontos e a presença de dor foi caracterizada quando a pontuação final era maior ou igual a três ${ }^{14}$.

Os outros sinais vitais como $\mathrm{FC}$, PAS, PAD, PAM e $\mathrm{SaO}_{2}$ foram identificados por meio monitorização digital (Omnimed, Omni 610). A FR foi obtida pela contagem das incursões respiratórias durante um minuto, já a TAX foi aferida através do sensor de temperatura corporal presente na incubadora.

Em seguida, os RN foram submetidos ao posicionamento em prono, sendo utilizados coxins em forma de rolos de tecido para elevar o tórax e facilitar a dinâmica diafragmática. A cabeça do RN foi lateralizada e alinhada com o tronco, posicionando os membros superiores e inferiores em flexão e as mãos próximas à face ${ }^{15}$. Este posicionamento durou 40 minutos e, em seguida, houve outra verificação dos sinais vitais em estudo. Após esta primeira intervenção, ocorreram os procedimentos de rotina da UTIN e os RN foram novamente estimulados pela equipe de saúde. Posteriormente, realizou-se outra vez a avaliação dos sinais vitais e seguiu-se com o posicionamento nas redes de descanso, também conhecidas como hammock: técnica em que o recém-nascido simula a posição uterina com sua natureza flexora e alinhada, suspenso pela redinha colocada na incubadora sem contato com colchão15, permitindo o padrão de flexão simulando a postura intrauterina. No entanto, foi necessário um rolo de tecido em nível do pescoço e da escápula para evitar a flexão excessiva do pescoço. Essa segunda intervenção também teve duração de 40 minutos. Por fim, houve outra verificação das variáveis de estudo. Assim sendo, a aplicação da escala NFCS e a verificação das outras 
variáveis referentes aos sinais vitais ocorreram em 4 momentos (antes e após cada procedimento de intervenção terapêutica). As redes de descanso foram confeccionadas em tecido tipo Brim $100 \%$ de algodão por se tratar de um material de baixo custo, alto capacidade de reutilização e fácil esterilização.

Os resultados obtidos foram comparados com o intuito de saber a eficiência e diferenças entre as intervenções. Os dados foram analisados estatisticamente sendo que as variáveis contínuas foram apresentadas como após esta primeira intervenção, ocorreram os procedimentos de rotina da UTIN e os RN foram novamente estimulados pela equipe de saúde. Posteriormente, realizou-se outra vez a avaliação dos sinais vitais e seguiu-se com o posicionamento nas redes de descanso, também conhecidas como hammock: técnica em que o recémnascido simula a posição uterina com sua natureza flexora e alinhada, suspenso pela redinha colocada na incubadora sem contato com colchão ${ }^{15}$, permitindo 0 padrão de flexão simulando a postura intrauterina. No entanto, foi necessário um rolo de tecido em nível do pescoço e da escápula para evitar a flexão excessiva do pescoço. Essa segunda intervenção também teve duração de 40 minutos. Por fim, houve outra verificação das variáveis de estudo. Assim sendo, a aplicação da escala NFCS e a verificação das outras variáveis referentes aos sinais vitais ocorreram em 4 momentos (antes e após cada procedimento de intervenção terapêutica). As redes de descanso foram confeccionadas em tecido tipo Brim $100 \%$ de algodão por se tratar de um material de baixo custo, alto capacidade de reutilização e fácil esterilização.

Os resultados obtidos foram comparados com o intuito de saber a eficiência e diferenças entre as intervenções. Os dados foram analisados estatisticamente sendo que as variáveis contínuas foram apresentadas como média e desvio-padrão, enquanto que as categóricas como frequências relativas e absolutas. A normalidade das variáveis foi testada por meio do teste de Lilliefors. As comparações intragrupo foram realizadas por meio do teste $\mathrm{t}$ para amostras repetidas, enquanto que as comparações intergrupo foram realizadas por meio do teste $t$ para amostras independentes. Para todas as análises, adotou-se um valor de alfa igual a $5 \%$ e o processamento dos dados foi realizado do pacote estatístico SPSS, versão 17.0 (Chicago, IL, EUA).

\section{Resultados}

Foram acompanhados 20 RNPT na UTIN, sendo 7 (35\%) do sexo masculino e 13 (65\%) do sexo feminino, tendo como motivo de internação síndrome do desconforto respiratório (SDR) com proporção de 18 (90\%) e 2 (10\%) com broncodisplasia, sabendo que a intervenção dos posicionamentos ocorreu no período de estabilidade do quadro clínico do paciente. A idade gestacional média foi de $32,00 \pm 2,94$ semanas. Quanto ao peso ao nascer observou-se uma média de $1932 \pm 630$ gramas, ou seja, 16 (80\%) nasceram com baixo peso $(<2500 \mathrm{~g})$ e $4(20 \%)$ com peso normal.

Nas Tabelas 1 e 2 foram analisados os sinais vitais e o escore de dor por meio da escala NFCS, antes e após o posicionamento prono e rede de descanso, respectivamente. Observaramse diferenças significativas nas comparações ao longo do tempo nas variáveis de $\mathrm{SaO}_{2}$ e dor. 
Tabela 1 - Análise dos sinais vitais e da dor antes e após o posicionamento em prono.

\begin{tabular}{lccccc}
\hline \multirow{2}{*}{ Variável } & \multicolumn{2}{c}{ Inicial } & \multicolumn{2}{c}{ Final } & p \\
\cline { 2 - 5 } & Média & DP & Média & DP & valor \\
\hline Temperatura $\left({ }^{\circ} \mathrm{C}\right)$ & 36,25 & 1,07 & 36,40 & 0,60 & 0,451 \\
Frequência Cardíaca $(\mathrm{bpm})$ & 133,10 & 10,08 & 127,65 & 10,50 & 0,067 \\
Frequência Respiratória $(\mathrm{rpm})$ & 49,90 & 9,85 & 49,25 & 7,89 & 0,694 \\
Saturação de Oxigênio $(\%)$ & 93,65 & 3,01 & 96,15 & 2,30 & $0,013^{*}$ \\
Pressão arterial média $(\mathrm{mmHg})$ & 52,30 & 15,05 & 53,20 & 15,95 & 0,302 \\
Pressão arterial sistólica $(\mathrm{mmHg})$ & 80,30 & 18,87 & 81,20 & 19,55 & 0,283 \\
Pressão arterial diastólica $(\mathrm{mmHg})$ & 41,35 & 6,28 & 41,60 & 8,77 & 0,834 \\
NCFS (escore) & 3,65 & 0,74 & 0,60 & 0,68 & $0,015^{\star}$ \\
\hline
\end{tabular}

${ }^{\circ} \mathrm{C}$ : grau Celsius; bpm: Batimentos por minutos; rpm: Respiração por minuto; mmHg: Milímetro de mercúrio; NFCS: Sistema de Codificação da Atividade Facial Neonatal; DP: Desvio-padrão; *Diferença significativa (teste t para amostras pareadas, $p<0,05$ ).

Tabela 2 - Análise dos sinais vitais e da dor antes e após as redes de descanso.

\begin{tabular}{lccccc}
\hline \multirow{2}{*}{ Variável } & \multicolumn{2}{c}{ Inicial } & \multicolumn{2}{c}{ Final } & $\mathbf{p}$ \\
\cline { 2 - 5 } & Média & DP & Média & DP & valor \\
\hline Temperatura $\left({ }^{\circ} \mathrm{C}\right)$ & 36,30 & 0,73 & 36,35 & 0,67 & 0,721 \\
Frequência Cardíaca $(\mathrm{bpm})$ & 131,75 & 10,92 & 128,85 & 10,46 & 0,222 \\
Frequência Respiratória $(\mathrm{rpm})$ & 52,85 & 8,92 & 51,50 & 8,18 & 0,463 \\
Saturação de Oxigênio $(\%)$ & 93,30 & 3,37 & 96,20 & 2,75 & $0,014^{*}$ \\
Pressão arterial média $(\mathrm{mmHg})$ & 55,00 & 16,68 & 52,95 & 14,36 & 0,265 \\
Pressão arterial sistólica $(\mathrm{mmHg})$ & 83,40 & 19,05 & 81,70 & 18,00 & 0,223 \\
Pressão arterial diastólica $(\mathrm{mmHg})$ & 42,80 & 10,91 & 41,75 & 7,58 & 0,582 \\
NCFS & 3,45 & 0,82 & 0,15 & 0,36 & $0,010^{*}$ \\
\hline
\end{tabular}

${ }^{\circ} \mathrm{C}$ : grau Celsius; bpm: Batimentos por minutos; rpm: Respiração por minuto; mmHg: Milímetro de mercúrio; NFCS: Sistema de Codificação da Atividade Facial Neonatal; DP: Desvio-padrão; *Diferença significativa (teste t para amostras pareadas, $p<0,05$ ).

Ao comparar as diferenças iniciais e finais para cada variável entre as intervenções prono e redes de descanso não se observou significância estatística, indicando que as duas intervenções causaram efeitos semelhantes nos sinais vitais e na dor nos RN, conforme evidenciado na Tabela 3.

\section{Discussão}

A amostra foi constituída basicamente por prematuros de baixo peso $(<2500 \mathrm{~g})$, com idade gestacional média de 32 semanas, tendo como principal diagnóstico SDR, refletindo o perfil dos pacientes atendidos na UTIN estudada. Características essas que 
foram também observadas por Nettina ${ }^{16}$, em que os prematuros com peso inferior a $2000 \mathrm{~g}$ e com idade gestacional entre 28 e 37 semanas apresentavam maior predisposição para desenvolver SDR.

A SDR do RN decorre da deficiência do surfactante alveolar, diretamente relacionada à imaturidade estrutural dos pulmões ${ }^{17}$. É caracterizada como uma das patologias mais frequentes do RNPT, prolongando, assim, o tempo de internação hospitalar e limitando seu prognóstico. Porém através do tratamento intensivo precoce é possível reverter o quadro clínico ${ }^{18}$.

Tabela 3. Comparação entre o posicionamento prono e redes de descanso.

\begin{tabular}{lccccc}
\hline \multirow{2}{*}{ Variável } & \multicolumn{2}{c}{ Rede (final - inicial) } & \multicolumn{2}{c}{ Prono (final - inicial) } & p \\
\cline { 2 - 5 } & Média & DP & Média & DP & valor \\
\hline Temperatura $\left({ }^{\circ} \mathrm{C}\right)$ & 0,05 & 0,60 & 0,15 & 0,88 & 0,541 \\
Frequência Cardíaca $(\mathrm{bpm})$ & $-2,90$ & 10,18 & $-5,45$ & 11,32 & 0,512 \\
Frequência Respiratória $(\mathrm{rpm})$ & $-1,35$ & 8,07 & $-0,65$ & 7,18 & 0,773 \\
Saturação de Oxigênio $(\%)$ & 2,90 & 3,45 & 2,50 & 2,33 & 0,694 \\
Pressão arterial média $(\mathrm{mmHg})$ & $-2,05$ & 7,87 & 0,90 & 3,80 & 0,142 \\
Pressão arterial sistólica $(\mathrm{mmHg})$ & $-1,70$ & 5,99 & 0,90 & 3,58 & 0,103 \\
Pressão arterial diastólica $(\mathrm{mmHg})$ & $-1,05$ & 8,28 & 0,25 & 5,09 & 0,562 \\
NCFS & $-3,30$ & 0,92 & $-3,05$ & 1,00 & 0,411 \\
\hline
\end{tabular}

${ }^{\circ} \mathrm{C}$ : grau Celsius; bpm: Batimentos por minutos; rpm: Respiração por minuto; mmHg: Milímetro de mercúrio; NFCS: Sistema de Codificação da Atividade Facial Neonatal; DP: Desvio-padrão; Sem diferença significativa (teste t para amostras independentes, $\mathrm{p}>$ $0,05)$.

Ainda que o número de nascimentos prematuros cresça a cada dia no país, tem se observado o aumento das chances de sobrevida dos mesmos $^{19,20}$. Esses resultados correspondem diretamente ao desenvolvimento do aparato tecnológico existente hoje nas UTIN, no entanto, paralelamente ao aprimoramento dos recursos terapêuticos, houve aumento do número de intervenções invasivas e dolorosas realizadas em busca de garantir a sobrevivência dos neonatos ${ }^{21}$.

Santos et al. ${ }^{1}$ explanaram que os bebês tendem a manifestar dor através de mudanças significativas em diferentes órgãos e sistemas, tendo como exemplo no sistema cardiovascular, com 0 aumento da pressão arterial e da FC e no sistema respiratório com a queda de $\mathrm{SaO}_{2}$ e elevação do consumo de oxigênio, além de variáveis comportamentais como choro, mímica facial e alteração do sono e vigília.

No presente estudo, quando avaliado o posicionamento prono e os seus efeitos sobre os sinais vitais e alívio da dor em RNPT, observou-se melhora da $\mathrm{SaO}_{2}$ e diminuição da dor quando comparados os valores antes e após o posicionamento, não observando diferenças significativas nas demais variáveis avaliadas. Quanto ao método redes de descanso, pode-se observar a 
melhora na $\mathrm{SaO}_{2}$ e a diminuição da dor quando comparados os valores de antes e depois do posicionamento. Nesse sentido, o estudo de Costa ${ }^{22}$ analisou os efeitos da postura na oxigenação arterial através da $\mathrm{SaO}_{2}, \mathrm{FC}$ e FR em neonatos posicionados em decúbito ventral (prono) e em hammock (redes de descanso). Os $\mathrm{RN}$ foram mantidos por 20 minutos em cada posição e foi visto que ao posicionar os prematuros em prono não se observou nenhuma mudança significativa nos parâmetros verificados. Em compensação, quando analisados nas redes de descanso houve redução da FC nos primeiros 15 minutos se mantendo até $0 \mathrm{fim}$ do procedimento, não apresentando diferenças significativas nas demais variáveis analisadas antes e após posicionamentos.

No presente estudo, após posicionar os $\mathrm{RN}$ em prono por 40 minutos, foi possível verificar a aumento da $\mathrm{SaO}_{2}$ e redução da dor. Malagoli et al. ${ }^{11}$, demonstra em seu estudo que a posição prono auxiliou no aumento $\mathrm{SaO}_{2}$, sugerindo maior eficácia do diafragma durante sua contração, melhorando a ventilação e otimizando as trocas gasosas. Por sua vez, Brunherotti e Martinez $^{23}$ descrevem que esse posicionamento estabiliza a caixa torácica e reduz a incoordenação toracoabdominal. No estudo em questão, ao avaliar os RNPT submetidos a 40 minutos no método redes de descanso, foi observado o aumento significativo da $\mathrm{SaO}_{2}$ e redução do escore de dor após o mesmo. Fato que corrobora com 0 estudo de Ziade et al. ${ }^{24}$ em que o uso da rede proporcionou, além da estimulação sensorial adequada por meio do balanço e conforto, a melhora na $\mathrm{FC}$ e $\mathrm{SaO}_{2}$, otimizando dessa forma 0 desenvolvimento neuropsicomotor do RNPT. Em contrapartida, o estudo realizado por Costa $^{24}$ fez uso de 20 minutos no posicionamento hammock e obteve redução da FC, porém não se identificou diferenças significativas na FR e na $\mathrm{SaO}_{2}$ quando comparados ao antes e ao após intervenção.

No que diz respeito à população do estudo quando comparados o método hammock e a posição prono quanto ao posicionamento que traria maiores benefícios aos RNPT no alívio da dor, não foi verificada diferença significativa uma vez que ambos se demonstraram eficazes na redução da dor em RNPT estáveis. Dados estes que não puderam ser comparados com outros estudos devido a ausência de literatura que cite a utilização dos posicionamentos como método para se obter o alívio da dor sobre os mesmos.

\section{Conclusão}

Foi possível identificar que 0 posicionamento em prono e rede de descanso promove melhora na $\mathrm{SaO}_{2}$ e na dor. Esses achados alertam a importância das mudanças de decúbito anteriormente citados em RNPT estáveis internados em UTIN por se tratarem de métodos não invasivos e de baixo custo que proporcionam benefícios para o seu desenvolvimento e minimizam os prejuízos resultantes da exposição ao estresse. 


\section{Referências}

1. Santos LM, Pereira MP, Santos LF, et al. Avaliação da dor no recém-nascido prematuro em Unidade de Terapia Intensiva. Revista Brasileira de Enfermagem. 2012;65(1):27-33.

2. Pessoa TA, Martins CB, Lima FC, Gaíva MA. O crescimento e desenvolvimento frente à prematuridade e baixo peso ao nascer. Avances em Enfermagem. 2015;33(3):401-11.

3. Cordeiro RA, Costa R. Métodos não farmacológicos para alívio do desconforto e da dor no recém-nascido: uma construção coletiva da enfermagem. Texto \& Contexto - Enfermagem. 2014;23(1):185-92.

4. Oliveira BB, Fontes DA, Oliveira MG. Dor em neonatos durante a assistência fisioterapêutica. Revista Unilus Ensino e Pesquisa. 2015;12(28):101-4.

5. Alves FB, Fialho FA, Dias IM, Amorim TM, Salvador M. Dor neonatal: a percepção da equipe de enfermagem na unidade de terapia intensiva neonatal. Revista Cuidarte. 2013;4(1):1-5.

6. Motta GC, Cunha ML. Prevenção e manejo não farmacológico da dor no recém-nascido. Revista Brasileira de Enfermagem. 2015;68(1):131-5.

7. Santos MC, Gomes MF, Capellini VK, Carvalho VC. Avaliação materna da dor em recém-nascidos prematuros. Revista da Rede de Enfermagem do Nordeste. 2015;15(6):842-7.

8. Bezerra IF, Torres VB, Lopes JM, Baroni MP, Pereira SA. Assessment of the influence of the hammock on neuromotor development in nursing full-term infantis. Journal of Human Growth and Development. 2014;24(1):106-111.

9. Costa KS. Redinhas de descanso e ninho em prematuros: ensaio clínico randomizado. [Dissertação, Mestrado em Enfermagem] Brasília: Universidade de Brasília; 2016.

10. Dourado FP, Oliveira NR, Campos IR. Posicionamento de prono na melhora da função respiratória de recém-nascidos pré-termos: uma revisão de literatura. Revista Movimenta. 2015;8(3):303-12.

11. Malagoli RC, Santos FF, Oliveira EA, Bouzada MC. Influência da posição prona na oxigenação, frequência respiratória e na força muscular nos recém-nascidos pré-termo em desmame da ventilação mecânica. Revista Paulista de Pediatria. 2012;30(2):251-6.

12. Prado C, Vale L. Fisioterapia Neonatal e Pediátrica. Barueri: Manole; 2012.

13. Numa AH, Hammer J, Newth CJ. Effect of Prone and Supine Positions on Functional Residual Capacity, Oxygenation, and Respiratory Mechanics in Ventilated Infants and Children. American Journal of Respiratory Care Medicine. 1997;156:1185-9.

14. Vignochi C, Teixeira PP, Nader SS. Efeitos da fisioterapia aquática na dor e no estado de sono e vigília de recémnascidos pré-termos estáveis internados em unidade de terapia intensiva neonatal. Revista Brasileira de Fisioterapia. 2010;14(3):214-20.

15. Costa KS, Beleza LO, Souza LM, Ribeiro LM. Rede de descanso e ninho: comparação entre efeitos fisiológicos e comportamentais. Revista Gaúcha de Enfermagem. 2016:37(esp):e62554.

16. Nettina SM. Pratica de enfermagem: distúrbios respiratórios pediátrico. $7^{\underline{a}}$ edição. Rio de Janeiro: Guanabara Koogan; 2003.

17. Nascimento Junior FJ, Silva JV, Ferreira AL, Rodrigues AP. A síndrome do desconforto respiratório do recémnascido: fisiopatologia e desafios assistenciais. Cadernos de Graduação Ciências Biológicas e da Saúde. 2014;2(2):189-98.

18. Santana SM, Novais MA, Zucchi P. Internações hospitalares de neonatos com síndrome do desconforto respiratório e sua participação nas internações hospitalares no âmbito do sistema único de saúde em 2015. International Journal of Health Management Review. 2016;2(1):1-18. 
19. Araújo FL, Manzo BF, Costa AC, Corrêa AR, Marcatto JO, Simão DA. Adesão ao bundle de inserção de cateter venoso central em unidades neonatais e pediátricas. Revista da Escola de Enfermagem da USP. 2017;51:e03269.

20. Santos RR, Petresco S, Morais BG, Manzolli PP, Mota DM, Santos N. Achados comportamentais e cognitivos em crianças de 5 a 11 anos nascidas com baixo peso. Revista da AMRIGS. 2014;58(3):203-8.

21. Nazareth CD, Lavor MF, Sousa TM. Ocorrência de dor em bebês internados em unidade de terapia intensiva neonatal de maternidade terciária. Revista de Medicina da UFC. 2015;55(1):33-7.

22. Costa AA. Estudo comparativo de prematuros posicionados em Hammock (redinhas) e decúbito ventral [internet]. Disponível em: http://interfisio.com.br/?artigo\&ID=153\&url $=$ Estudo-comparativ

-de-prematuros-posicionados-em-Hammock-(Redinhas)-e--decubito-ventral. Acesso: 15 de fevereiro de 2018.

23. Brunherotti MA, Martinez FE. Response of oxygen saturation in preterm infants receiving rib cage stabilization with an elastic band in two body positions: a randomized clinical trial. Brazilian Journal of Physical Therapy. 2013;12(2):105-111.

24. Ziade S, Toledo M, Rebelo C. No embalo da rede. Revista Eletrônica Minas Saúde. 2009;2(2):20-3. 